\title{
On the quasi-arithmetic Gauss-type iteration
}

\author{
PaWę PAsteczKaid
}

\begin{abstract}
For a sequence of continuous, monotone functions $f_{1}, \ldots, f_{n}: I \rightarrow \mathbb{R}(I$ is an interval) we define the mapping $M: I^{n} \rightarrow I^{n}$ as a Cartesian product of quasi-arithmetic means generated by $f_{j}$-s. It is known that, for every initial vector, the iteration sequence of this mapping tends to the diagonal of $I^{n}$. We will prove that whenever all $f_{j}$-s are $\mathcal{C}^{2}$ with nowhere vanishing first derivative, then this convergence is quadratic. Furthermore, the limit $\frac{\operatorname{Var} M^{k+1}(v)}{\left(\operatorname{Var} M^{k}(v)\right)^{2}}$ will be calculated in a nondegenerated case.
\end{abstract}

Mathematics Subject Classification. 26E60, 26B15, 26A18, 39B12.

Keywords. Gaussian iteration process, Invariant means, Quasi-arithmetic means, Iteration, Mean, Mean-type mapping.

\section{Introduction}

In 1800 (this year is due to [33]) Gauss introduced the arithmetic-geometric mean as a limit in the following two-term recursion:

$$
x_{k+1}=\frac{x_{k}+y_{k}}{2}, \quad y_{k+1}=\sqrt{x_{k} y_{k}},
$$

where $x_{0}=x$ and $y_{0}=y$ are two positive parameters. Gauss [14, p. 370] proved that both $\left(x_{k}\right)_{k=1}^{\infty}$ and $\left(y_{k}\right)_{k=1}^{\infty}$ converge to a common limit, which is called the arithmetic-geometric mean of the initial values $x_{0}$ and $y_{0}$. Borwein and Borwein [6] extended some earlier ideas [13,22,32] and generalized this iteration to a vector of continuous, strict means of an arbitrary length.

Invariant means in a family of quasi-arithmetic means were studied by many authors, for example Burai [7], Daróczy-Páles [9], Jarczyk [17] and Jarczyk and Matkowski [18]. In fact invariant means were extensively studied in recent years, see for example the papers by Baják-Páles [2-5], by Daróczy-Páles $[8,10,11]$, by Głazowska $[15,16]$, by Matkowski [23-26], by Matkowski-Páles [27], and by the author [30]. 
Recall that for a given interval $I$, a mean defined on $I$ is any function $\mathcal{M}: \bigcup_{n=1}^{\infty} I^{n} \rightarrow I$ such that $\min (a) \leq \mathcal{M}(a) \leq \max (a)$ for every admissible $a$. The mean is strict if $\min (a)<\mathcal{M}(a)<\max (a)$ unless $a$ is a constant vector.

It is known, [6, Theorem 8.2], that for all twice continuously differentiable, strict means $\mathcal{M}, \mathcal{N}$ and sequences

$$
x_{k+1}=\mathcal{M}\left(x_{k}, y_{k}\right), \quad y_{k+1}=\mathcal{N}\left(x_{k}, y_{k}\right), \quad k \in \mathbb{N}_{+} \cup\{0\},
$$

the difference $\left|x_{k}-y_{k}\right|$ tends to zero quadratically for all $x_{0}=x$ and $y_{0}=y$.

Following [6, section 8.7], we will consider the iteration of multidimensional means. Given a natural number $n \in \mathbb{N}$ and a vector of means $\left(\mathcal{M}_{1}, \ldots, \mathcal{M}_{n}\right)$ defined on a common interval $I$, let us define the mapping $\mathbf{M}: I^{n} \rightarrow I^{n}$ by

$$
\mathbf{M}(a):=\left(\mathcal{M}_{1}(a), \ldots, \mathcal{M}_{n}(a)\right), \quad a \in I^{n} .
$$

Whenever for every $i \in\{1, \ldots, N\}$ the limit of its iteration sequence $\lim _{k \rightarrow \infty}\left[\mathbf{M}^{k}(a)\right]_{i}$ exists and does not depend on $i$, we call it the invariant mean of $\left(\mathcal{M}_{i}\right)$ and denote it by $\mathcal{M}_{\otimes}(a)$. Some authors refers to $\mathcal{M}_{\otimes}(a)$ as Gaussian product. Indeed, $\mathcal{M}_{\otimes}$ can be characterized as a unique mean satisfying the equality $\mathcal{M}_{\otimes} \circ \mathbf{M}=\mathcal{M}_{\otimes}$ (cf. e.g. Matkowski [23]). He also proved that whenever all means are continuous and strict then $\mathcal{M}_{\otimes}$ is a uniquely defined continuous and strict mean.

Some special case is that for some $k_{0} \in \mathbb{N}$ the vector $\mathbf{M}^{k_{0}}(a)$ is constant. Then, for all $k \geq k_{0}$, we have $\mathbf{M}^{k}(a)=\mathbf{M}^{k_{0}}(a)$. In particular each entry of this vector equals $\mathcal{M}_{\otimes}(a)$. If it is the case for some nonconstant vector $a$, then we will call such an iteration process degenerated. It can be easily verified that under some mild condition regarding the comparability of means an iteration process is never degenerated. Such results are however outside the scope of this paper and are omitted.

Gauss' iteration process in a case when all means are quasi-arithmetic will be of our interest. It was already under investigation in [30]. We are going to continue the research in this area. In particular we will prove the multidimensional counterpart of [6, Theorem 8.2] in a case when all considered means are quasi-arithmetic. Furthermore we will show that, under some conditions, not only the convergence is quadratic, but also the characteristic ratio is closely related to the so-called Arrow-Pratt index.

\section{Quasi-arithmetic means}

Quasi-arithmetic means were introduced in a series of nearly simultaneous papers in the early 1930s $[12,20,29]$ as a generalization of the already mentioned family of power means. For a continuous and strictly monotone function $f: I \rightarrow \mathbb{R}\left(I\right.$ is an interval) and a vector $a=\left(a_{1}, a_{2}, \ldots, a_{n}\right) \in I^{n}, n \in \mathbb{N}$ we define 


$$
\mathcal{A}_{f}(a):=f^{-1}\left(\frac{f\left(a_{1}\right)+f\left(a_{2}\right)+\cdots+f\left(a_{n}\right)}{n}\right) .
$$

It is easy to verify that for $I=\mathbb{R}_{+}$and $f=\pi_{p}$, where $\pi_{p}(x):=x^{p}$ if $p \neq 0$ and $\pi_{0}(x):=\ln x$, then the mean $\mathcal{A}_{f}$ coincides with the $p$-th power mean (from now on denoted by $\mathcal{P}_{p}$ ); this fact had already been noticed by Knopp [19] before quasi-arithmetic means were formally introduced.

In the course of dealing with the Gaussian iteration process we will use the notation of the Arrow-Pratt index [1,31], which was also investigated by Mikusiński [28]. Whenever $f: I \rightarrow \mathbb{R}$ is twice differentiable with nowhere vanishing first derivative we can define the operator $P_{f}:=f^{\prime \prime} / f^{\prime}$. It can be proved that the comparability of quasi-arithmetic means is equivalent to the pointwise comparability of the respective Arrow-Pratt indexes (see [28] for details).

Following the idea from [30] we will assume that all the considered functions are smooth enough to apply the operator $P$. Moreover, for technical reasons, we assume that the second derivative is of almost bounded variation (finite variation restricted to every compact interval; cf. [21, p. 135]). Using this definition we introduce the class

$$
\mathcal{S}(I):=\left\{f \in \mathcal{C}^{2}(I): f^{\prime} \neq 0 \text { and } f^{\prime \prime} \text { is of almost bounded variation }\right\} .
$$

Obviously, as $f^{\prime} \neq 0$, each element belonging to $\mathcal{S}(I)$ is a continuous and strictly monotone function, and therefore it generates a quasi-arithmetic mean. The assumption that $f^{\prime \prime}$ is of almost bounded variation is technical, however important from the point of view of the present paper (this is also the setting which was extensively used in the previous paper [30]).

Following the idea from [30] we are going to deal with the Gaussian iteration of quasi-arithmetic means. Define, for the vector $\mathbf{f}=\left(f_{j}\right)_{j=1}^{n}$ of continuous, strictly monotone functions on $I$, the mapping $\mathbf{A}_{\mathbf{f}}: I^{n} \rightarrow I^{n}$ by

$$
\mathbf{A}_{\mathbf{f}}(a):=\left(\mathcal{A}_{f_{1}}(a), \ldots, \mathcal{A}_{f_{n}}(a)\right) .
$$

In fact $\mathbf{A}_{\mathbf{f}}$ is the quasi-arithmetic counterpart of the function $\mathbf{M}$, which appears in the definition of invariant mean. Then it is known that there exists a unique continuous and strict mean $\mathcal{A}_{\otimes}: I^{n} \rightarrow I$ such that $\mathcal{A}_{\otimes} \circ \mathbf{A}_{\mathbf{f}}=\mathcal{A}_{\otimes}$. It also has further implications but let us introduce some necessary notations first. For a vector $a$ of real numbers we denote its arithmetic mean, variance, and spread briefly by $\bar{a}, \operatorname{Var}(a)$, and $\delta(a):=\max (a)-\min (a)$, respectively.

It is known that for every vector $a \in I^{n}$, the sequence $\left(\operatorname{Var}\left(\mathbf{A}_{\mathbf{f}}^{k}(a)\right)\right)_{k \in \mathbb{N}}$ tends to zero. Moreover, due to [30], if $\mathbf{f} \in \mathcal{S}(I)^{n}$ then this convergence is double exponential with fractional base. We will prove that, in a non-degenerated case, this sequence tends to zero quadratically and, moreover, we will calculate the limit

$$
\lim _{k \rightarrow \infty} \frac{\operatorname{Var}\left(\mathbf{A}_{\mathbf{f}}^{k+1}(a)\right)}{\operatorname{Var}\left(\mathbf{A}_{\mathbf{f}}^{k}(a)\right)^{2}} .
$$




\subsection{Approximate value of quasi-arithmetic means}

We are now heading towards the calculation of quasi-arithmetic means in the spirit of Taylor. In fact the crucial identity was already established in the previous paper. Let us recall this result (Riemann-Stieltjes integral is used in its wording).

Lemma 2.1. ([30], Lemma 4.1) For every $f \in \mathcal{S}(I)$ and $a \in I^{n}, n \in \mathbb{N}$,

$$
\mathcal{A}_{f}(a)=\bar{a}+\frac{1}{2} \operatorname{Var}(a) \cdot P_{f}(\bar{a})+R_{f}(a)+S_{f}(a),
$$

where

$$
\begin{aligned}
R_{f}(a) & :=\frac{1}{2 n \cdot f^{\prime}(\bar{a})} \cdot \sum_{i=1}^{n} \int_{\bar{a}}^{a_{i}}\left(a_{i}-t\right)^{2} d f^{\prime \prime}(t), \\
S_{f}(a) & :=\int_{\bar{a}}^{\mathcal{A}_{f}(a)} \frac{\left(f(u)-f\left(\mathcal{A}_{f}(a)\right)\right) f^{\prime \prime}(u)}{f^{\prime}(u)^{2}} d u .
\end{aligned}
$$

It was also proved [30, Lemma 4.2] that

$$
\begin{aligned}
\left|R_{f}(a)\right| & \leq \frac{1}{6 n} \cdot \exp \left(\left\|P_{f}\right\|_{*}\right) \cdot \sum_{i=1}^{n}\left|a_{i}-\bar{a}\right|^{3}, \\
\left|S_{f}(a)\right| & \leq\left(\mathcal{A}_{f}(a)-\bar{a}\right)^{2} \cdot \exp \left(\left\|P_{f}\right\|_{*}\right),
\end{aligned}
$$

where the $*$-norm is defined as $\|g\|_{*}:=\sup _{a, b \in \operatorname{dom}(g)}\left|\int_{a}^{b} g(t) d t\right|$.

What was not noticed is that if the second derivative of $f$ is locally Lipschitz then the error terms can be majorized much more efficiently. We are going to prove this in a while. First, define $\mathcal{S}^{\text {Lip }}(I):=\{f \in$ $\mathcal{S}(I): f^{\prime \prime}$ is locally Lipschitz $\} ; \mathcal{S}_{K}(I):=\left\{f \in \mathcal{S}(I):\left\|P_{f}\right\|_{\infty} \leq K\right\}$ for $K>0$ and $\mathcal{S}_{K}^{\text {Lip }}(I):=\mathcal{S}^{\text {Lip }}(I) \cap \mathcal{S}_{K}(I)$.

For the purpose of this estimation let us make the purely technical assumption $K=1$, which will be omitted soon.

Lemma 2.2. For every $f \in \mathcal{S}_{1}^{\text {Lip }}(I)$ and $a \in I^{n}, n \in \mathbb{N}$,

$\left|R_{f}(a)\right| \leq \frac{\operatorname{Lip}\left(f^{\prime \prime}\right)}{2\left|f^{\prime}(\bar{a})\right|} \cdot \delta(a) \operatorname{Var}(a) \quad$ and $\quad\left|S_{f}(a)\right| \leq \frac{\alpha^{2}}{4} \exp \left(\left\|P_{f}\right\|_{*}\right) \delta(a)^{4}$,

where $\alpha:=\frac{3+7 e}{3}$.

Proof. By the mean-value theorem there exist $\xi_{1}, \ldots, \xi_{n}, \eta \in(\min a, \max a)$ such that

$$
\begin{aligned}
R_{f}(a) & =\frac{1}{2 n \cdot f^{\prime}(\bar{a})} \cdot \sum_{i=1}^{n} \int_{\bar{a}}^{a_{i}}\left(a_{i}-t\right)^{2} d f^{\prime \prime}(t) \\
& =\frac{1}{2 n \cdot f^{\prime}(\bar{a})} \cdot \sum_{i=1}^{n}\left(-\left(a_{i}-\bar{a}\right)^{2} f^{\prime \prime}(\bar{a})-2 \int_{\bar{a}}^{a_{i}}\left(a_{i}-t\right) f^{\prime \prime}(t) d t\right)
\end{aligned}
$$




$$
\begin{aligned}
& =\frac{1}{2 n \cdot f^{\prime}(\bar{a})} \cdot \sum_{i=1}^{n}\left(a_{i}-\bar{a}\right)^{2}\left(f^{\prime \prime}\left(\xi_{i}\right)-f^{\prime \prime}(\bar{a})\right) \\
& =\frac{1}{2 n} \cdot \sum_{i=1}^{n}\left(a_{i}-\bar{a}\right)^{2} \frac{f^{\prime \prime}(\eta)-f^{\prime \prime}(\bar{a})}{f^{\prime}(\bar{a})}=\frac{\operatorname{Var}(a)}{2} \cdot \frac{f^{\prime \prime}(\eta)-f^{\prime \prime}(\bar{a})}{f^{\prime}(\bar{a})} .
\end{aligned}
$$

Therefore

$$
\left|R_{f}(a)\right|=\frac{|\eta-\bar{a}| \operatorname{Var}(a)}{2\left|f^{\prime}(\bar{a})\right|} \cdot\left|\frac{f^{\prime \prime}(\eta)-f^{\prime \prime}(\bar{a})}{\eta-\bar{a}}\right| \leq \frac{\operatorname{Lip}\left(f^{\prime \prime}\right)}{2\left|f^{\prime}(\bar{a})\right|} \cdot \delta(a) \operatorname{Var}(a) .
$$

We will now prove the second inequality. By (2.1), we have

$$
\left|S_{f}(a)\right| \leq\left(\mathcal{A}_{f}(a)-\bar{a}\right)^{2} \cdot \exp \left(\left\|P_{f}\right\|_{*}\right) .
$$

Furthermore, by [30, Lemma 4.3], we get $\left|\mathcal{A}_{f}(a)-\bar{a}\right| \leq \frac{\alpha}{2} \delta(a)^{2}$. Thus

$$
\left|S_{f}(a)\right| \leq \frac{\alpha^{2}}{4} \exp \left(\left\|P_{f}\right\|_{*}\right) \delta(a)^{4},
$$

what was to be proved.

\section{Main result}

Binding the two results above we can establish the main theorem of the present note. In order to make the notation more compact the brief sum-type notation of means will be used (that is we will write $\mathcal{M}_{k=1}^{n}\left(t_{k}\right)$ instead of $\mathcal{M}\left(t_{1}, \ldots, t_{n}\right)$ ). Additionally, for the same reason, we will use the \pm notation of the remainder (with the natural interpretation).

Theorem 3.1. Let $I$ be an interval, $K>0, n \in \mathbb{N},\left(f_{j}\right)_{j=1}^{n} \in \mathcal{S}_{K}^{L i p}(I)^{n}$, and a be a vector having entries in $I$. Then

$$
\operatorname{Var}\left(\boldsymbol{A}_{\mathbf{f}}(a)\right)=\frac{1}{4} \operatorname{Var}(a)^{2} \operatorname{Var}\left(P_{\mathbf{f}}(\bar{a})\right) \pm 4 C K^{5} \delta(a)^{5} \pm\left(3 C^{2}+C_{2}^{2}\right) K^{6} \delta(a)^{6},
$$

where $P_{\mathbf{f}}: I \rightarrow \mathbb{R}^{n}$ is defined by $P_{\mathbf{f}}(x):=\left(P_{f_{1}}(x), \ldots, P_{f_{n}}(x)\right), \alpha:=\frac{3+7 e}{3}$, and

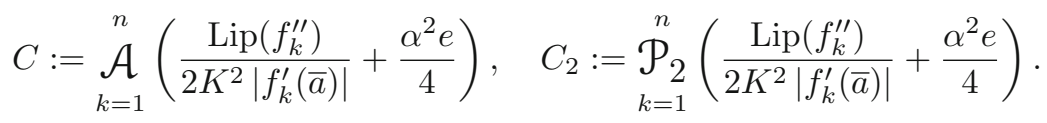

Recall that $\mathcal{A}$ and $\mathcal{P}_{2}$ stand for arithmetic and quadratic means, respectively.

Proof. Applying the machinery described in [30, section 4.1] we can apply the mapping

$$
\mathcal{S}_{K}^{L i p}(I) \ni f(x) \mapsto f(x / K) \in \mathcal{S}_{1}^{L i p}(K \cdot I)
$$


to each function $f_{k}$. Therefore we will assume, without loss of generality, that $K=1$. In fact to make such an assumption possible, we need to verify that the statement in the theorem in both setups are equivalent. Precise calculations are not very simple, but rather straightforward.

In the case when $\delta(a) \geq 1$ we have $C \geq \alpha^{2} e / 4>1$, thus the admissible error on the right hand side is at least $3 \delta(a)^{6}$. Meanwhile

$$
\begin{aligned}
\left|\operatorname{Var}\left(\mathbf{A}_{\mathbf{f}}(a)\right)-\frac{1}{4} \operatorname{Var}(a)^{2} \operatorname{Var}\left(P_{\mathbf{f}}(\bar{a})\right)\right| & \leq \operatorname{Var}\left(\mathbf{A}_{\mathbf{f}}(a)\right)+\frac{1}{4} \operatorname{Var}(a)^{2} \operatorname{Var}\left(P_{\mathbf{f}}(\bar{a})\right) \\
& \leq \delta(a)^{2}+\frac{\delta(a)^{4}}{4} \leq \frac{5}{4} \delta(a)^{4} \leq \frac{5}{4} \delta(a)^{6} .
\end{aligned}
$$

From now on we will assume that $\delta(a)<1$. By Lemmas 2.1 and 2.2,

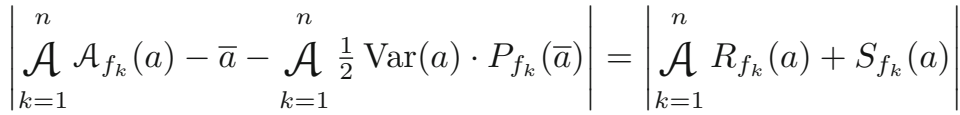

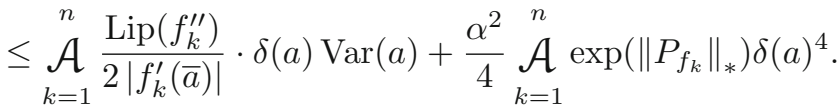

We know that $\operatorname{Var}(a) \leq \delta(a)^{2}$, thus we obtain

$$
\begin{aligned}
\underset{k=1}{\mathcal{A}} \mathcal{A}_{f_{k}}(a)= & \bar{a}+\underset{k=1}{\mathcal{A}} \frac{1}{2} \operatorname{Var}(a) \cdot P_{f_{k}}(\bar{a}) \pm \underset{k=1}{\operatorname{A}} \frac{\operatorname{Lip}\left(f_{k}^{\prime \prime}\right)}{2\left|f_{k}^{\prime}(\bar{a})\right|} \cdot \delta(a)^{3} \\
& \pm \frac{\alpha^{2}}{4} \underset{k=1}{\mathcal{A}} \exp \left(\left\|P_{f_{k}}\right\|_{*}\right) \delta(a)^{4} .
\end{aligned}
$$

As $\delta(a)<1$ we get $\delta(a)^{4} \leq \delta(a)^{3}$ and $\exp \left(\left\|P_{f_{k}}\right\|_{*}\right) \leq e$. Therefore

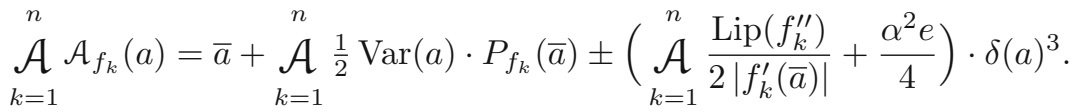

We can express it briefly as

$$
\underset{k=1}{n} \mathcal{A}_{f_{k}}(a)=\bar{a}+\mathcal{A}_{k=1}^{n} \frac{1}{2} \operatorname{Var}(a) \cdot P_{f_{k}}(\bar{a}) \pm C \delta(a)^{3} .
$$

Thus, using Lemmas 2.1 and 2.2 again, we have

$$
\begin{aligned}
\mathcal{A}_{f_{j}}(a)-\mathcal{A}_{k=1}^{n} \mathcal{A}_{f_{k}}(a)= & \frac{1}{2} \operatorname{Var}(a) \cdot\left(P_{f_{j}}(\bar{a})-\underset{k=1}{\mathcal{A}_{f_{k}}}(\bar{a})\right) \\
& \pm\left(C+\frac{\operatorname{Lip}\left(f_{j}^{\prime \prime}\right)}{2\left|f_{j}^{\prime}(\bar{a})\right|}+\frac{\alpha^{2} e}{4}\right) \delta(a)^{3} .
\end{aligned}
$$


Therefore

$$
\begin{aligned}
\left(\mathcal{A}_{f_{j}}(a)\right. & \left.-\underset{k=1}{\mathcal{A}} \mathcal{A}_{f_{k}}(a)\right)^{2}=\frac{1}{4} \operatorname{Var}(a)^{2} \cdot\left(P_{f_{j}}(\bar{a})-\underset{k=1}{\mathcal{A}} P_{f_{k}}(\bar{a})\right)^{2} \\
& \pm\left(C+\frac{\operatorname{Lip}\left(f_{j}^{\prime \prime}\right)}{2\left|f_{j}^{\prime}(\bar{a})\right|}+\frac{\alpha^{2} e}{4}\right) \delta(a)^{3} \operatorname{Var}(a) \cdot\left|P_{f_{j}}(\bar{a})-\underset{k=1}{\mathcal{A}} P_{f_{k}}(\bar{a})\right| \\
& \pm\left(C+\frac{\operatorname{Lip}\left(f_{j}^{\prime \prime}\right)}{2\left|f_{j}^{\prime}(\bar{a})\right|}+\frac{\alpha^{2} e}{4}\right)^{2} \delta(a)^{6} .
\end{aligned}
$$

But, by $\left|P_{f_{k}}\right| \leq 1$ we get $\left|P_{f_{j}}(\bar{a})-\mathcal{A}_{k=1}^{n} P_{f_{k}}(\bar{a})\right| \leq 2$, moreover $\operatorname{Var}(a) \leq \delta(a)^{2}$. Whence

$$
\begin{aligned}
\left(\mathcal{A}_{f_{j}}(a)\right. & \left.-\underset{k=1}{\mathcal{A}} \mathcal{A}_{f_{k}}(a)\right)^{2}=\frac{1}{4} \operatorname{Var}(a)^{2} \cdot\left(P_{f_{j}}(\bar{a})-\underset{k=1}{\mathcal{A}} P_{f_{k}}(\bar{a})\right)^{2} \\
& \pm 2 \cdot\left(C+\frac{\operatorname{Lip}\left(f_{j}^{\prime \prime}\right)}{2\left|f_{j}^{\prime}(\bar{a})\right|}+\frac{\alpha^{2} e}{4}\right) \delta(a)^{5} \pm\left(C+\frac{\operatorname{Lip}\left(f_{j}^{\prime \prime}\right)}{2\left|f_{j}^{\prime}(\bar{a})\right|}+\frac{\alpha^{2} e}{4}\right)^{2} \delta(a)^{6} .
\end{aligned}
$$

We now apply the operator $\mathcal{A}_{j=1}^{n}$ side-by-side to the equality above to obtain

$$
\begin{aligned}
\operatorname{Var}\left(\mathbf{A}_{\mathbf{f}}(a)\right) & =\frac{1}{4} \operatorname{Var}(a)^{2} \operatorname{Var}\left(P_{\mathbf{f}}(\bar{a})\right) \pm \underset{j=1}{\mathcal{A}}\left(2 C+\frac{\operatorname{Lip}\left(f_{j}^{\prime \prime}\right)}{\left|f_{j}^{\prime}(\bar{a})\right|}+\frac{\alpha^{2} e}{2}\right) \delta(a)^{5} \\
& \pm \underset{j=1}{\mathcal{A}}\left(C+\frac{\operatorname{Lip}\left(f_{j}^{\prime \prime}\right)}{2\left|f_{j}^{\prime}(\bar{a})\right|}+\frac{\alpha^{2} e}{4}\right)^{2} \delta(a)^{6} .
\end{aligned}
$$

But

$$
\underset{j=1}{\mathcal{A}}\left(2 C+\frac{\operatorname{Lip}\left(f_{j}^{\prime \prime}\right)}{\left|f_{j}^{\prime}(\bar{a})\right|}+\frac{\alpha^{2} e}{2}\right)=2 C+\underset{j=1}{\mathcal{A}}\left(\frac{\operatorname{Lip}\left(f_{j}^{\prime \prime}\right)}{\left|f_{j}^{\prime}(\bar{a})\right|}+\frac{\alpha^{2} e}{2}\right)=4 C .
$$

Additionally

$$
\begin{aligned}
\underset{j=1}{\mathcal{A}}(C+ & \left.\frac{\operatorname{Lip}\left(f_{j}^{\prime \prime}\right)}{2\left|f_{j}^{\prime}(\bar{a})\right|}+\frac{\alpha^{2} e}{4}\right)^{2}=C^{2}+2 C \cdot \underset{j=1}{\mathcal{A}}\left(\frac{\operatorname{Lip}\left(f_{j}^{\prime \prime}\right)}{2\left|f_{j}^{\prime}(\bar{a})\right|}+\frac{\alpha^{2} e}{4}\right) \\
& +\underset{j=1}{\mathcal{A}}\left(\frac{\operatorname{Lip}\left(f_{j}^{\prime \prime}\right)}{2\left|f_{j}^{\prime}(\bar{a})\right|}+\frac{\alpha^{2} e}{4}\right)^{2}=C^{2}+2 C^{2}+C_{2}^{2}=3 C^{2}+C_{2}^{2} .
\end{aligned}
$$

Binding (3.2), (3.3), and (3.4) we obtain the final statement.

Remark. As the values of $f_{j}$-s outside the interval $[\min a, \max a]$ do not affect the left hand side of the inequality (3.1), we can simply assume that $I=[\min a, \max a]$ i.e. take a Lipschitz constant on the restricted domain only. 
Corollary 3.2. Let $\mathbf{f}=\left(f_{1}, \ldots, f_{n}\right) \in \mathcal{S}^{\text {Lip }}(I)^{n}$ and $a \in I^{n}$. Consider the mapping $\boldsymbol{A}_{\mathbf{f}}:=\left(\mathcal{A}_{f_{1}}, \ldots, \mathcal{A}_{f_{n}}\right): I^{n} \rightarrow I^{n}$. Then either the iteration process $\boldsymbol{A}_{\mathbf{f}}$ is degenerated or

$$
\lim _{k \rightarrow \infty} \frac{\operatorname{Var} \boldsymbol{A}_{\mathbf{f}}^{k+1}(a)}{\left(\operatorname{Var} \boldsymbol{A}_{\mathbf{f}}^{k}(a)\right)^{2}}=\frac{\operatorname{Var}\left(P_{\mathbf{f}}\left(\mathcal{A}_{\otimes}(a)\right)\right)}{4} .
$$

Proof. Assume that the iteration process is not degenerated. Applying the machinery described in [30, section 4 .1] we can assume that $\mathbf{f} \in \mathcal{S}_{1}^{\text {Lip }}(I)^{n}$. We know that

$$
\operatorname{Var}(a) \in\left(\delta(a)^{2} / 2 n, \delta(a)^{2}\right) .
$$

Thus, if we divide (3.1) side-by-side by $\operatorname{Var}(a)^{2}$ we get

$$
\frac{\operatorname{Var}\left(\mathbf{A}_{\mathbf{f}}(a)\right)}{\operatorname{Var}(a)^{2}}=\frac{1}{4} \operatorname{Var}\left(P_{\mathbf{f}}(\bar{a})\right) \pm 16 n^{2} C \delta(a) \pm 4 n^{2}\left(3 C^{2}+C_{2}^{2}\right) \delta(a)^{2} .
$$

If we now put $a \leftarrow \mathbf{A}_{\mathbf{f}}^{k}(a)$, we obtain

$$
\begin{aligned}
\frac{\operatorname{Var}\left(\mathbf{A}_{\mathbf{f}}^{k+1}(a)\right)}{\operatorname{Var}\left(\mathbf{A}_{\mathbf{f}}^{k}(a)\right)^{2}}=\frac{1}{4} & \operatorname{Var}\left(P_{\mathbf{f}}\left(\overline{\mathbf{A}_{\mathbf{f}}^{k}(a)}\right)\right) \pm 16 n^{2} C \delta\left(\mathbf{A}_{\mathbf{f}}^{k}(a)\right) \\
& \pm 4 n^{2}\left(3 C^{2}+C_{2}^{2}\right) \delta\left(\mathbf{A}_{\mathbf{f}}^{k}(a)\right)^{2} .
\end{aligned}
$$

But we know that $\delta\left(\mathbf{A}_{\mathbf{f}}^{k}(a)\right) \rightarrow 0$ and $\mathbf{A}_{\mathbf{f}}^{k}(a) \rightarrow\left(\mathcal{A}_{\otimes}(a), \ldots, \mathcal{A}_{\otimes}(a)\right)$ as $k \rightarrow \infty$. Therefore

$$
\lim _{k \rightarrow \infty} \frac{\operatorname{Var}\left(\mathbf{A}_{\mathbf{f}}^{k+1}(a)\right)}{\operatorname{Var}\left(\mathbf{A}_{\mathbf{f}}^{k}(a)\right)^{2}}=\frac{\operatorname{Var}\left(P_{f}\left(\mathcal{A}_{\otimes}(a)\right)\right)}{4},
$$

which concludes the proof.

By the property (3.5) we also obtain

Corollary 3.3. Let $\mathbf{f}=\left(f_{1}, \ldots, f_{n}\right) \in \mathcal{S}^{\text {Lip }}(I)^{n}$ and $a \in I^{n}$. Consider a mapping $\boldsymbol{A}_{\mathbf{f}}:=\left(\mathcal{A}_{f_{1}}, \ldots, \mathcal{A}_{f_{n}}\right): I^{n} \rightarrow I^{n}$. Then either the iteration process $\left(\boldsymbol{A}_{\mathbf{f}}^{k}(a)\right)_{k=1}^{\infty}$ is degenerated or $\left(\delta\left(\boldsymbol{A}_{\mathbf{f}}^{k}(a)\right)\right)_{k=1}^{\infty}$ tends to zero quadratically.

Open Access. This article is distributed under the terms of the Creative Commons Attribution 4.0 International License (http://creativecommons.org/licenses/by/4.0/), which permits unrestricted use, distribution, and reproduction in any medium, provided you give appropriate credit to the original author(s) and the source, provide a link to the Creative Commons license, and indicate if changes were made.

\section{References}

[1] Arrow, K.J.: Aspects of the Theory of Risk-Bearing. Yrjö Jahnsson Foundation, Helsinki (1965) 
[2] Baják, Sz, Páles, Zs: Computer aided solution of the invariance equation for two-variable Gini means. Comput. Math. Appl. 58, 334-340 (2009)

[3] Baják, Sz, Páles, Zs: Invariance equation for generalized quasi-arithmetic means. Aequ. Math. 77, 133-145 (2009)

[4] Baják, Sz, Páles, Zs: Computer aided solution of the invariance equation for two-variable Stolarsky means. Appl. Math. Comput. 216(11), 3219-3227 (2010)

[5] Baják, Sz, Páles, Zs: Solving invariance equations involving homogeneous means with the help of computer. Appl. Math. Comput. 219(11), 6297-6315 (2013)

[6] Borwein, J.M., Borwein, P.B.: Pi and the AGM: A Study in the Analytic Number Theory and Computational Complexity. Wiley, New York (1987)

[7] Burai, P.: A Matkowski-Sutô type equation. Publ. Math. Debr. 70, 233-247 (2007)

[8] Daróczy, Z.: Functional equations involving means and Gauss compositions of means. Nonlinear Anal. 63(5-7), e417-e425 (2005)

[9] Daróczy, Z., Páles, Zs.: A Matkowski-Sutô type problem for quasi-arithmetic means of order $\alpha$. In: Daróczy, Z., Páles, Zs, editors. Functional Equations-Results and Advances, vol. 3 of Adv. Math. (Dordr.), pp. 189-200. Kluwer Acad. Publ., Dordrecht (2002)

[10] Daróczy, Z., Páles, Zs: Gauss-composition of means and the solution of the MatkowskiSutô problem. Publ. Math. Debr. 61(1-2), 157-218 (2002)

[11] Daróczy, Z., Páles, Zs: The Matkowski-Sutô problem for weighted quasi-arithmetic means. Acta Math. Hungar. 100(3), 237-243 (2003)

[12] de Finetti, B.: Sul concetto di media. Giornale dell' Instituto, Italiano degli Attuarii 2, 369-396 (1931)

[13] Foster, D.M.E., Phillips, G.M.: The arithmetic-harmonic mean. Math. Comput. 42(165), 183-191 (1984)

[14] Gauss, C.F.: Nachlass: Aritmetisch-geometrisches Mittel. In: Werke 3 (Göttingem 1876), pp. 357-402. Königliche Gesellschaft der Wissenschaften (1818)

[15] Głazowska, D.: A solution of an open problem concerning Lagrangian mean-type mappings. Cent. Eur. J. Math. 9(5), 1067-1073 (2011)

[16] Głazowska, D.: Some Cauchy mean-type mappings for which the geometric mean is invariant. J. Math. Anal. Appl. 375(2), 418-430 (2011)

[17] Jarczyk, J.: Invariance of weighted quasi-arithmetic means with continuous generators. Publ. Math. Debr. 71(3-4), 279-294 (2007)

[18] Jarczyk, J., Matkowski, J.: Invariance in the class of weighted quasi-arithmetic means. Ann. Polon. Math. 88(1), 39-51 (2006)

[19] Knopp, K.: Über Reihen mit positiven Gliedern. J. Lond. Math. Soc. 3, 205-211 (1928)

[20] Kolmogorov, A.N.: Sur la notion de la moyenne. Rend. Accad. dei Lincei 6(12), 388-391 (1930)

[21] Kuczma, M., Choczewski, B., Ger, R.: Iterative functional equations. In: Encyclopedia of Mathematics and its Applications, vol. 32. Cambridge University Press, Cambridge (1990)

[22] Lehmer, D.H.: On the compounding of certain means. J. Math. Anal. Appl. 36, 183-200 (1971)

[23] Matkowski, J.: Iterations of mean-type mappings and invariant means. Ann. Math. Sil. 13:211-226 (1999). European Conference on Iteration Theory (Muszyna-Złockie, 1998)

[24] Matkowski, J.: On iteration semigroups of mean-type mappings and invariant means. Aequ. Math. 64(3), 297-303 (2002)

[25] Matkowski, J.: Lagrangian mean-type mappings for which the arithmetic mean is invariant. J. Math. Anal. Appl. 309(1), 15-24 (2005)

[26] Matkowski, J.: Iterations of the mean-type mappings and uniqueness of invariant means. Ann. Univ. Sci. Bp. Sect. Comput. 41, 145-158 (2013)

[27] Matkowski, J., Páles, Zs: Characterization of generalized quasi-arithmetic means. Acta Sci. Math. (Szeged) 81(3-4), 447-456 (2015) 
[28] Mikusiński, J.G.: Sur les moyennes de la forme $\psi^{-1}\left[\sum q \psi(x)\right]$. Studia Math. 10(1), 90-96 (1948)

[29] Nagumo, M.: Über eine Klasse der Mittelwerte. Jpn. J. Math. 7, 71-79 (1930)

[30] Pasteczka, P.: Iterated quasi-arithmetic mean type mappings. Colloq. Math. 144(2), 215-228 (2016)

[31] Pratt, J.W.: Risk aversion in the small and in the large. Econometrica 32(1/2), 122-136 (1964)

[32] Schoenberg, I.J.: Mathematical Time Exposures. Mathematical Association of America, Washington, DC (1982)

[33] Toader, G., Toader, S.: Greek means and the arithmetic-geometric mean. In: RGMIA Monographs. Victoria University (2005)

Paweł Pasteczka

Institute of Mathematics

Pedagogical University of Cracow

Podchorążych str. 2

30-084 Kraków

Poland

e-mail: pawel.pasteczka@up.krakow.pl

Received: January 23, 2018

Revised: April 17, 2018 